\title{
Comparison Study of Image Compression with Walsh Wavelets
}

\author{
Surbhi Saxena \\ M.Tech Student IET Alwar, (Raj)
}

\author{
Deepak Chaudhary \\ Assistant Professor, CSE, IET Alwar (Raj
}

\begin{abstract}
Now a days storage space and running time of algorithm or execution time for computers are critical and challenge aspect, With the purpose of decrease the cost of storage pace for images lot of methods are available, researchers have focused on optimization methodology or techniques for comparison, There are many techniques for optimization but here main focus is on wavelets based techniques with other transformation as a hybrid to find the optimal solutions for image compression. The overall objective of performing a set of instruction with wavelets to identify the impact of wavelets algorithms on optimization approaches by scope, performance and cost. This Self Literature Review is conducted on more than 20 articles, and find the algorithms used in each approach and group them according to their similarities.
\end{abstract}

\section{Keywords}

image compression, wavelets, storage, etc.

\section{INTRODUCTION}

Image compression problem is going to be crucial to the growth of multimedia. In other words, it is the natural technology for handling the increased spatial purposes of today's imaging sensors and evolving broadcast television standards.

Now a day, the usage of digital image or applications of image processing in various disciplines is growing rapidly. Video and image transmission is becoming digital and more and more digital image sequences are used in multimedia applications. A digital image is a collection of pixels, which can be look like a small dots on the screen and it becomes more complex when the pixels of image are colored means it is divided into components which is depend upon nature of image

For the analysis of some medical or other images for compression, the literature survey has been done and discussed. There are many medical image compressions using hybrid methodology or techniques are evolving every day in research. Hence, it is mandatory to study theoretically about it, to understand the methodology also to use the best methods during compression of medical image [1-5].

\section{LITERATURE SURVEY}

Er. Ramandeep Kaur, Navneet Randhawa [9] (2012) describes architecture of discrete cosine Transformation and Discrete wavelets Transformation standard of an image compression. the image size without losing much of the resolutions computed can be done by DWT and the values less than prespecified threshold. In this paper also covers some basic upbringings of wavelet analysis, data compression and proposed how DCT and DWT can be used for image compression in a single pipe line to solve a particular problem i.e. image compression. And in the last reconstruction concept was discuss for hybrid algorithm.
Nikita Bansal, Sanjay Kumar Dubey [10] (2013) has also proposed a scheme for Image Compression using same transformation DCT and DWT which is called as hybrid compression technique. The aim of DCT is high compaction property and often requires less computational resources and DWT is use for multi resolution transformation. The goal of this paper is to achieve maximum image compression ratio with preserving quality of decompressed image.

Manjinder Kaur,GaganpreetKaur [11] (2013)describes in him/her paper to analyze lossless image compression techniques, through which it offered high compression ratio and concluded that if someone wants more compression ratio than go for Lossy compression and if original image are to be identical than go for lossless compression.

Akshay Kekre, Dr. Sanjay Pokle [12] (2013) describes the image compression algorithm based on two transformation i.e. wavelet transform and differential pulse code modulation (DPCM). The model show the best result as compared to the single wavelet transform technique. This conclusion comes on the basis of measuring compression ratio and PSNR.

Neelesh Kumar Sahu,ChandrashekharKamargaonkar [13] (2013) studied different compression techniques. In this paper authors discussed compression methods such as Optimized Volume of Interest, JPEG-LS, Interface Coding, Motion Compensation and Customized Entropy Coding. EZW Encoding with Huffman Encoder, Curve let Transform, Visually Lossless Compression, and Simple Selective Scan Order with Bit Plane Slicing on the basis of compression ratio and compression quality.

Navpreet Saroya, Prabhpreet Kaur [14] (2014) proposed a hybrid theory based on Discrete Cosine Transform (DCT) and Discrete Wavelet transforms (DWT) and implemented in MATLAB because these are lossy techniques. The aim of this paper at compression using DCT and Wavelet Transform by selecting proper method to improve the result of quality parameters like for PSNR (Peak Signal to Noise Ratio) has been obtained.

R. Bhavithra, L. Ayeesha Begame, K.S.L. Deepika [15] (2014) purposed a survey which is based on image compression under different image compression techniques using different mathematical transformation. Motive and need to improve image compression for professional fields such as medical imaging. In Medical fields compression is necessary for huge data storage and data transfer for diagnosis. On the basis of this analysis any one can conclude that the advantages of compression and choosing correct method for compression.

Malvika Dixit, Harbinder Singh [16] (2014) proposed a theory based on Discrete Wavelet Transform (DWT) and Vector quantization for compression and implemented in MATLAB. A new image compression theme gives the high compression ratio with no considerable degradation of image quality. 
Mahinderpal Singh, Meenakshi Garg [17] (2014) proposed a combined approached (DWT-DCT) used in image compression. The property of DCT has high energy compaction and also requires less computational resources. On the other word, DWT is multi resolution Transformation. In this paper, proposed mixed (DWT-DCT) algorithm for image compression and reconstruction taking benefit from the advantages of both algorithms.

K. Ayyappa Swamy [18] (2015) proposed image compression technique using Discrete Cosine Transform (DCT) in which different quantization laves is used to observe different compression ratio and different peak signal-to-noise ratio. In DWT, with different levels and different thresholds we can observe different compression ratio and different peak signalto-noise ratio. Based on the application we can select the quantization level. When comparing DCT with DWT, results of DWT are better than DCT. In this paper we are proposing a new hybrid transform by combining DCT and DWT which gives better compression ratio for same PSNR. In proposed transform first image is compressed using DWT and the approximation coefficients of compressed image are again compressed using DCT.

As the name suggests, Wavelets are small time limited waves having zero average value. Different types of available wavelets are shown in Table. These wavelets are the basis function for wavelet analysis. Types of Mother Wavelets Different wavelet families and corresponding mother wavelets are tabulated below. Following wavelets in the last column of the table indicate a wavelet being a part of an infinite family of wavelets.

Table 1: Types of wavelets

\begin{tabular}{|c|c|c|c|}
\hline $\begin{array}{l}\text { Sr. } \\
\text { No }\end{array}$ & $\begin{array}{l}\text { Mother } \\
\text { wavelet } \\
\text { family } \\
\text { names }\end{array}$ & $\begin{array}{l}\text { Abbrevi } \\
\text { ations }\end{array}$ & Wavelets \\
\hline 1 & Haar & Haar & \\
\hline 2 & $\begin{array}{l}\text { Daubechi } \\
\text { es }\end{array}$ & $\mathrm{Db}$ & $\begin{array}{l}\text { db1 db2 db3 db4 db5 } \\
\text { db6 db7 db8 } \\
\text { db9 db10 db** }\end{array}$ \\
\hline 3 & Symlets & Sym & $\begin{array}{l}\text { sym2 sym3 sym4 sym5 } \\
\text { sym6 sym7 sym8 } \\
\text { sym** }\end{array}$ \\
\hline 4 & Coiflets & Coif & $\begin{array}{l}\text { coif1 coif2 coif3 } \\
\text { coif4 coif5 }\end{array}$ \\
\hline 5 & $\begin{array}{l}\text { BiorSplin } \\
\text { es }\end{array}$ & Bior & $\begin{array}{l}\text { bior } 1.1 \text { bior } 1.3 \text { bior } 1.5 \\
\text { bior } 2.2 \text { bior } 2.4 \text { bior } 2.6 \\
\text { bior } 2.8 \text { bior } 3.1 \text { bior } 3.3 \\
\text { bior } 3.5 \text { bior } 3.7 \text { bior } 3.9 \\
\text { bior } 4.4 \text { bior } 5.5 \\
\text { bior } 6.8\end{array}$ \\
\hline 6 & $\begin{array}{l}\text { ReverseB } \\
\text { ior }\end{array}$ & Rbio & $\begin{array}{l}\text { rbio1.1 rbio1.3 rbio1.5 } \\
\text { rbio2.2 rbio2.4 rbio2.6 } \\
\text { rbio2.8 rbio3.1 rbio3.3 } \\
\text { rbio3.5 rbio3.7 rbio3.9 } \\
\text { rbio4.4 rbio5.5 } \\
\text { rbio6.8 }\end{array}$ \\
\hline 7 & Meyer & Meyr & \\
\hline 8 & DMeyer & Dmey & \\
\hline 9 & Gaussian & Gaus & $\begin{array}{l}\text { gaus1 gaus2 gaus3 } \\
\text { gaus4 gaus5gaus6 gaus7 } \\
\text { gaus8 gaus** }\end{array}$ \\
\hline 10 & $\begin{array}{l}\text { Mexican } \\
\text { hat }\end{array}$ & Mexh & \\
\hline 11 & Morlet & Morl & \\
\hline 12 & $\begin{array}{l}\text { Complex } \\
\text { Gaussian }\end{array}$ & Gaus & $\begin{array}{l}\text { cgau 1cgau2 } 2 \text { cgau3c } \\
\text { gau } 4 \quad \text { cgau5 } \\
\text { cgau** }\end{array}$ \\
\hline 13 & Shannon & Shan & shan $1-1.5$ shan $1-1$ \\
\hline
\end{tabular}

\begin{tabular}{|c|c|c|c|}
\hline & & & $\begin{array}{ll}\text { shan1-0.5 } & \text { shan1- } \\
0.1 & \text { shan2-3 } \\
\text { shan** } & \\
\end{array}$ \\
\hline 14 & $\begin{array}{l}\text { Frequency } \\
\text { B-Spline }\end{array}$ & Fbsp & $\begin{array}{l}\text { fbsp1-1-1.5 fbsp1-1-1 } \\
\text { fbsp1-1-0.5 fbsp2-1-1 } \\
\text { fbsp2-1-0.5 fbsp2- } \\
1-0.1 \text { fbsp** }\end{array}$ \\
\hline 15 & $\begin{array}{l}\text { Complex } \\
\text { Morlet }\end{array}$ & Cmor & $\begin{array}{l}\text { cmor } 1-1.5 \text { cmor } 1-1 \\
\text { cmor } 1-0.5 \text { cmor } 1-0.1 \\
\text { cmor** }\end{array}$ \\
\hline
\end{tabular}

\section{COLOR IMAGE COMPRESSION ALGORITHMS}

Step 1: Read the Color Image

Step 2: Convert Color image RGB to Ycbcr

Step 3: Decide the name of Wavelets Family

Step 4: Apply function for compression for each layer independently $\mathrm{y}, \mathrm{cb}$ and $\mathrm{cr}$.

Step 5: Decide value of factor and parameter. Factor is used at the quantization for Low and High frequencies and parameter is used for reduce low-frequency quality to increase compression ratio.

Step 6: Function call (hybrid of Hadamard function and dwt2 coding ) for first layer of YCbcr image.

Step 7: Apply first and second level wavelets transformation decomposition.

Step 8: Apply Quantization and Walsh 2D- Transform

Step 9: Compress all data by using Arithmetic Coding

Step 10: Save information and compressed data in a Record..... "Header"

Step 12: Repeat step 6-10 for second and third layer of YCbcr image.

Step 11: Assign location for the compressed data and save with extension "*.sur".

\section{COLOR IMAGE DECOMPRESSION ALGORITHMS}

Step 1: Read compress data from the file

Step 2: Function call (hybrid of Hadamard function and dwt2 decoding) apply decompression on each header

Step 3: Using Arithmetic Decoding for extract original data

Step 4: Apply inverse Quantization and inverse Hadamard function Transform

Step 5: Inverse -Wavelet Transform Stage-1

Step 6: Inverse -Wavelet Transform Stage-2

Step 7: Repeat step 2-6 for second and third Header of compressed image

Step 8: Collect all layers in one matrix Ycbcr

Step 9: Convert $\mathrm{YCbCr}$ format to RGB

Step 10: Image save on hard disk with extension .bmp

\section{COMPRESSION TOOL}

All the above said algorithms have been implemented in MATLAB and design MATLAB too with the combination of different .m files. Here there is two buttons in compression 
tool, first button for compression and second for decompression. When we click on first button tool read one input image from disk space suppose Test1.bmp (Test1.bmp, Test2.bmp, Test 3.bmp, Test4.bmp and Test5.bmp) and compression start, in few second compression finishes and one compressed file with extension sur saved in current directory of MATLAB. Once we click on decompression tool read .sur file from current directory and decompression start in few second decompression finishes and new decompressed file with extension .bmp saved in current directory of MATLAB. And record the size of compressed image (extension .sur and decompressed image which is useful to calculate Compression ratio. This process is repeat for each member of wavelets family and result record in format of tabular for each wavelets.

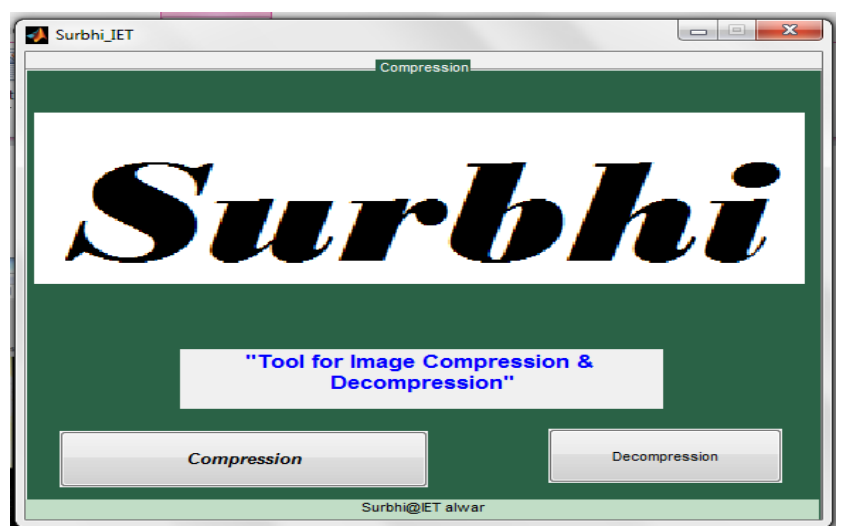

Fig1: Compression Tool

\section{Compression Ratio}

Compression ratio and PSNR Benchmarks in image data compression are the compression ratio and PSNR (Peak Signal to Noise Ratio). The compression ratio is used to measure the ability of data compression by comparing the size of the image being compressed to the size of the original image. The greater the compression ratio means the better the wavelet function. PSNR is one of the parameters that can be used to quantify image quality. PSNR parameter is often used as a benchmark level of similarity between reconstructed image and the original image. Larger PSNR will produce better image quality.

Given $\mathrm{n} 1$ and $\mathrm{n} 2$ denoting the information-carrying units in two data sets that represent the same information/image then ratio can be defined as $=1-\mathrm{n} 1 / \mathrm{n} 2$.

e.g suppose size of original image is $225 \mathrm{~kb}$ and size of compressed image is $11.2 \mathrm{~kb}$ then ratio can be expressed mathematically in this manner. $\mathrm{CR}=1-11.2 / 225=.9502$ but in $\% \mathrm{CR}=95.02 \%$, sometimes it can described in this manner i.e.

\section{Compression ratio $=(225) / 11.2$} $=20.089 / 1$
The interpretation is that 20.089 pixels of input image are expressed as 1 pixel in the output image.

\section{Saving Percentage $=1-1 / 20.089$ $=95.02 \%$}

In this paper there are five different input images (Test1.bmp, Test 2.bmp, Test3.bmp, Test 4.bmp and Test 5.bmp) with different size (225 KB, 768KB, $1.37 \mathrm{MB}, 2.25 \mathrm{MB}$ and 3.70 $\mathrm{MB}$ respectively) are process to record the compression ratio. And which is shown graphically in below table. All algorithms and results are implemented in MATLAB R2013a with system configuration intel ${ }^{\circledR}$ Core $^{\mathrm{TM}}$ i5-2410M CPU@2.30Ghz 4.00GB RAM 64-bit Operating System.

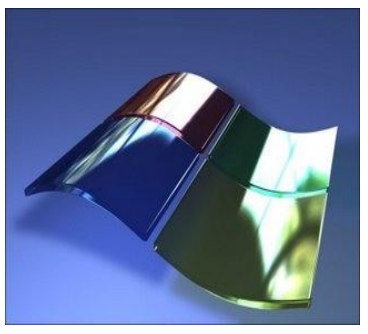

Test1.bmp

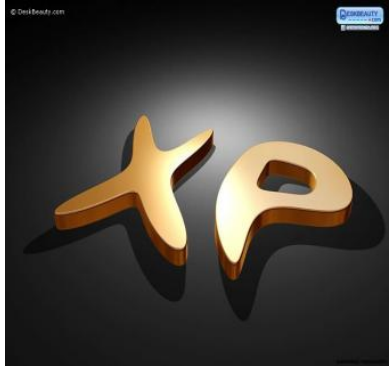

Test3.bmp

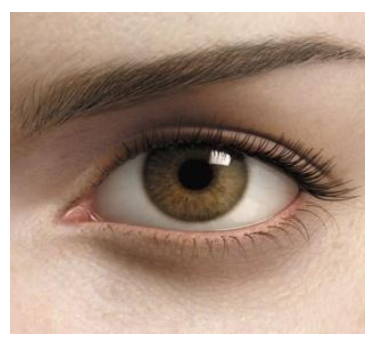

Test2.bmp

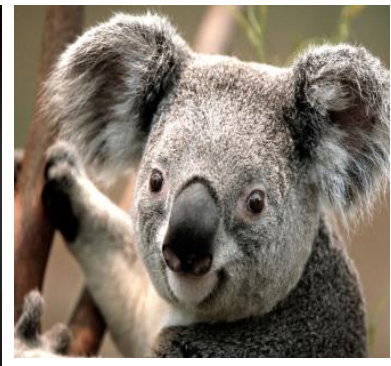

Test4.bmp

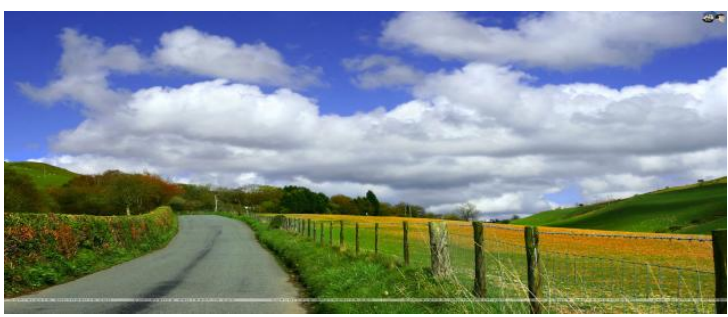

Test5.bmp
One success measurement in image data compression is the compression ratio. The compression ratio is to measure the ability of data compression by comparing the sizes of the image being compressed with the original size. The greater the compression ratio means the better the wave function-in short. The test results of the wavelet influence towards the compression ratio of several test images can be seen in table.

Table2: Compression Analysis for wavelets family 'bior'

\begin{tabular}{|c|c|c|c|c|c|c|c|c|}
\hline $\begin{array}{l}\text { SN. } \\
\text { O }\end{array}$ & $\begin{array}{l}\text { Input } \\
\text { Image }\end{array}$ & $\begin{array}{l}\text { Dimensio } \\
\text { n }\end{array}$ & $\begin{array}{l}\text { Origina } \\
\text { I Size }\end{array}$ & $\begin{array}{l}\text { Wavelet } \\
\mathrm{s}\end{array}$ & $\begin{array}{l}\text { Compresse } \\
\text { d Size }\end{array}$ & $\begin{array}{l}\text { Decompresse } \\
\text { d Size }\end{array}$ & $\begin{array}{l}\text { Decompresse } \\
\text { d Dimension }\end{array}$ & $\begin{array}{l}\text { Compressio } \\
\text { n Ratio }\end{array}$ \\
\hline \multirow{3}{*}{1} & \multirow{3}{*}{$\begin{array}{c}\text { Test1.bm } \\
\mathrm{p}\end{array}$} & \multirow{3}{*}{$320 * 240$} & \multirow{3}{*}{$225 \mathrm{~KB}$} & bior1.1 & $11.2 \mathrm{~KB}$ & $225 K B$ & $320 * 240$ & 95.022 \\
\hline & & & & bior1.3 & $12.8 \mathrm{~KB}$ & $225 \mathrm{~KB}$ & $320 * 240$ & 94.311 \\
\hline & & & & bior1.5 & $13.3 \mathrm{~KB}$ & $225 \mathrm{~KB}$ & $320 * 240$ & 94.088 \\
\hline \multirow[b]{2}{*}{2} & \multirow{2}{*}{ Tes2.bmp } & \multirow{2}{*}{$512 * 512$} & \multirow{2}{*}{$768 \mathrm{~KB}$} & bior1.1 & $22.3 \mathrm{~KB}$ & $768 \mathrm{~KB}$ & $512 * 512$ & 97.096 \\
\hline & & & & bior1.3 & $26.2 \mathrm{~KB}$ & $768 \mathrm{~KB}$ & $512 * 512$ & 96.588 \\
\hline
\end{tabular}




\begin{tabular}{|c|c|c|c|c|c|c|c|c|}
\hline & & & & bior1.5 & $26.8 \mathrm{~KB}$ & $768 \mathrm{~KB}$ & $512 * 512$ & 96.510 \\
\hline \multirow[b]{3}{*}{3} & \multirow{3}{*}{$\begin{array}{c}\text { Test3.bm } \\
\mathrm{p}\end{array}$} & \multirow{3}{*}{$800 * 600$} & \multirow{3}{*}{$1.37 \mathrm{MB}$} & bior1.1 & $27.1 \mathrm{~KB}$ & $1.37 \mathrm{MB}$ & $800 * 600$ & 98.068 \\
\hline & & & & bior1.3 & $29.8 K B$ & $1.37 \mathrm{MB}$ & $800 * 600$ & 97.8757 \\
\hline & & & & bior1.5 & $29.3 K B$ & $1.37 \mathrm{MB}$ & $800 * 600$ & 97.911 \\
\hline \multirow[b]{3}{*}{4} & \multirow{3}{*}{$\begin{array}{c}\text { Test4.bm } \\
\mathrm{p}\end{array}$} & \multirow{3}{*}{$1024 * 768$} & \multirow{3}{*}{$2.25 \mathrm{MB}$} & bior1.1 & $89.7 K B$ & $2.25 \mathrm{MB}$ & $1024 * 768$ & 96.106 \\
\hline & & & & bior1.3 & $101 \mathrm{~KB}$ & $2.25 \mathrm{MB}$ & $1024 * 768$ & 95.616 \\
\hline & & & & bior1.5 & $104 \mathrm{~KB}$ & $2.25 \mathrm{MB}$ & $1024 * 768$ & 95.486 \\
\hline \multirow[b]{3}{*}{5} & \multirow{3}{*}{$\begin{array}{c}\text { Test5.bm } \\
\mathrm{p}\end{array}$} & \multirow{3}{*}{$1440 * 900$} & \multirow{3}{*}{$3.70 \mathrm{MB}$} & bior1.1 & $150 \mathrm{~KB}$ & $3.70 \mathrm{MB}$ & $1440 * 900$ & 96.040 \\
\hline & & & & bior1.3 & $162 \mathrm{~KB}$ & $3.70 \mathrm{MB}$ & $1440 * 900$ & 95.7242 \\
\hline & & & & bior1.5 & $168 \mathrm{~KB}$ & $3.70 \mathrm{MB}$ & $1440 * 900$ & 95.5658 \\
\hline
\end{tabular}

Table 3: Compression Analysis for wavelets family 'coif'

\begin{tabular}{|c|c|c|c|c|c|c|c|c|}
\hline SN.O & $\begin{array}{l}\text { Input } \\
\text { Image }\end{array}$ & Dimension & $\begin{array}{l}\text { Original } \\
\text { Size }\end{array}$ & Wavelets & $\begin{array}{l}\text { Compressed } \\
\text { Size }\end{array}$ & $\begin{array}{l}\text { Decompressed } \\
\text { Size }\end{array}$ & $\begin{array}{l}\text { Decompressed } \\
\text { Dimension }\end{array}$ & $\begin{array}{l}\text { Compression } \\
\text { Ratio }\end{array}$ \\
\hline \multirow{4}{*}{1} & \multirow{4}{*}{ Test1.bmp } & \multirow{4}{*}{$320 * 240$} & \multirow{4}{*}{$225 \mathrm{~KB}$} & Coif1 & $12.0 \mathrm{~kb}$ & $225 \mathrm{~kb}$ & $320 * 240$ & 94.666 \\
\hline & & & & Coif2 & $11.4 \mathrm{~kb}$ & $228 \mathrm{~KB}$ & $322 * 242$ & 94.93 \\
\hline & & & & Coif3 & $12.3 \mathrm{~kb}$ & $225 \mathrm{~KB}$ & $320 * 240$ & 94.53 \\
\hline & & & & Coif4 & $14.0 \mathrm{~kb}$ & $228 \mathrm{~KB}$ & $322 * 242$ & 93.77 \\
\hline \multirow[b]{4}{*}{2} & \multirow{4}{*}{ Tes2.bmp } & \multirow{4}{*}{$512 * 512$} & \multirow{4}{*}{$768 \mathrm{~KB}$} & Coif1 & $24.2 \mathrm{~kb}$ & $768 \mathrm{~kb}$ & $512 * 512$ & 96.8489 \\
\hline & & & & Coif2 & $22.4 \mathrm{~kb}$ & $775 \mathrm{~KB}$ & $514 * 514$ & 97.08 \\
\hline & & & & Coif3 & $27.4 \mathrm{~kb}$ & $768 \mathrm{~KB}$ & $512 * 512$ & 96.432 \\
\hline & & & & Coif4 & $26.4 \mathrm{~kb}$ & $775 \mathrm{~KB}$ & $514 * 514$ & 96.56 \\
\hline \multirow[b]{4}{*}{3} & \multirow{4}{*}{ Test3.bmp } & \multirow{4}{*}{$800 * 600$} & \multirow{4}{*}{$1.37 \mathrm{MB}$} & Coif1 & $27.1 \mathrm{~kb}$ & $1.37 \mathrm{MB}$ & $800 * 600$ & 98.068 \\
\hline & & & & Coif2 & $22.4 \mathrm{~kb}$ & $1.38 \mathrm{MB}$ & $802 * 602$ & 98.40 \\
\hline & & & & Coif3 & $27.4 \mathrm{~kb}$ & $1.37 \mathrm{MB}$ & $800 * 600$ & 98.046 \\
\hline & & & & Coif4 & $29.2 \mathrm{~kb}$ & $1.38 \mathrm{MB}$ & $802 * 602$ & 97.91 \\
\hline \multirow[b]{4}{*}{4} & \multirow{4}{*}{ Test4.bmp } & \multirow{4}{*}{$1024 * 768$} & \multirow{4}{*}{$2.25 \mathrm{MB}$} & Coif1 & $93.2 \mathrm{~kb}$ & $2.25 \mathrm{MB}$ & $1024 * 768$ & 95.954 \\
\hline & & & & Coif2 & $91.9 \mathrm{~kb}$ & $2.26 \mathrm{MB}$ & $1026 * 770$ & 96.011 \\
\hline & & & & Coif3 & $95.6 \mathrm{~kb}$ & $2.25 \mathrm{MB}$ & $1024 * 768$ & 95.850 \\
\hline & & & & Coif4 & $100 \mathrm{~kb}$ & $2.26 \mathrm{MB}$ & $1026 * 770$ & 95.65 \\
\hline \multirow[b]{4}{*}{5} & \multirow{4}{*}{ Test5.bmp } & \multirow{4}{*}{$1440 * 900$} & \multirow{4}{*}{$3.70 \mathrm{MB}$} & Coif1 & $149.0 \mathrm{~kb}$ & $3.76 \mathrm{MB}$ & $1440 * 900$ & 96.06 \\
\hline & & & & Coif2 & $152.0 \mathrm{~kb}$ & $3.72 \mathrm{MB}$ & $1442 * 902$ & 95.98 \\
\hline & & & & Coif3 & $156 \mathrm{~kb}$ & $3.70 \mathrm{MB}$ & $1440 * 900$ & 95.882 \\
\hline & & & & Coif4 & $162 \mathrm{~kb}$ & $3.72 \mathrm{MB}$ & $1442 * 902$ & 95.72 \\
\hline
\end{tabular}

Table 4: Compression Analysis for wavelets family ' $d b$ '

\begin{tabular}{|c|c|c|c|c|c|c|c|c|}
\hline $\begin{array}{c}\text { SN. } \\
\text { O }\end{array}$ & $\begin{array}{l}\text { Input } \\
\text { Image }\end{array}$ & $\begin{array}{c}\text { Dimensio } \\
\mathbf{n}\end{array}$ & $\begin{array}{c}\text { Origina } \\
\text { I Size }\end{array}$ & $\begin{array}{c}\text { Wavelet } \\
\text { s }\end{array}$ & $\begin{array}{c}\text { Compresse } \\
\text { d Size }\end{array}$ & $\begin{array}{c}\text { Decompresse } \\
\text { d Size }\end{array}$ & $\begin{array}{l}\text { Decompresse } \\
\text { d Dimension }\end{array}$ & $\begin{array}{c}\text { Compressio } \\
\text { n Ratio }\end{array}$ \\
\hline \multirow{7}{*}{1} & \multirow{7}{*}{$\begin{array}{c}\text { Test1.bm } \\
\mathrm{p}\end{array}$} & \multirow{7}{*}{$320 * 240$} & \multirow{7}{*}{$225 \mathrm{~KB}$} & $\mathrm{db} 1$ & $11.2 \mathrm{~KB}$ & $225 K B$ & $320 * 240$ & 95.022 \\
\hline & & & & $\mathrm{db} 2$ & $11.5 \mathrm{~KB}$ & $228 \mathrm{~KB}$ & $322 * 242$ & 94.88 \\
\hline & & & & $\mathrm{db} 3$ & $11.8 \mathrm{~KB}$ & $225 \mathrm{~KB}$ & $320 * 240$ & 94.755 \\
\hline & & & & $\mathrm{db} 4$ & $12.3 \mathrm{~KB}$ & $228 \mathrm{~KB}$ & $322 * 242$ & 94.53 \\
\hline & & & & $\mathrm{db} 5$ & $11.9 \mathrm{~KB}$ & $225 \mathrm{~KB}$ & $320 * 240$ & 94.711 \\
\hline & & & & Db6 & $11.3 \mathrm{~kb}$ & $228 \mathrm{~kb}$ & $322 * 242$ & 94.97 \\
\hline & & & & Db7 & $12.9 \mathrm{~KB}$ & $225 \mathrm{~KB}$ & $320 * 240$ & 94.266 \\
\hline \multirow[b]{7}{*}{2} & \multirow{7}{*}{ Tes2.bmp } & \multirow{7}{*}{$512 * 512$} & \multirow{7}{*}{$768 \mathrm{~KB}$} & $\mathrm{db} 1$ & $22.3 \mathrm{~KB}$ & $768 \mathrm{~KB}$ & $512 * 512$ & 97.096 \\
\hline & & & & $\mathrm{db} 2$ & $22.7 \mathrm{~KB}$ & $775 \mathrm{~KB}$ & $514 * 514$ & 97.04 \\
\hline & & & & $\mathrm{db} 3$ & $24.0 K B$ & $768 \mathrm{~KB}$ & $512 * 512$ & 96.875 \\
\hline & & & & $\mathrm{db} 4$ & $23.8 \mathrm{~KB}$ & $775 K B$ & $514 * 514$ & 96.90 \\
\hline & & & & $\mathrm{db} 5$ & $22.4 \mathrm{~KB}$ & $775 \mathrm{~KB}$ & $512 * 512$ & 97.08 \\
\hline & & & & Db6 & $22.4 \mathrm{~kb}$ & $775 \mathrm{~kb}$ & $514 * 514$ & 97.08 \\
\hline & & & & $\mathrm{Db} 7$ & $24.6 \mathrm{~KB}$ & $768 \mathrm{~KB}$ & $512 * 512$ & 93.79 \\
\hline \multirow[b]{5}{*}{3} & \multirow{5}{*}{$\begin{array}{c}\text { Test3.bm } \\
\mathrm{p}\end{array}$} & \multirow{5}{*}{$800 * 600$} & \multirow{5}{*}{$1.37 \mathrm{MB}$} & $\mathrm{db} 1$ & $27.4 \mathrm{~KB}$ & $1.37 \mathrm{MB}$ & $800 * 600$ & 98.003 \\
\hline & & & & $\mathrm{db} 2$ & $26.1 \mathrm{~KB}$ & $1.38 \mathrm{MB}$ & $802 * 602$ & 98.06 \\
\hline & & & & $\mathrm{db3}$ & $27.1 \mathrm{~KB}$ & $1.37 \mathrm{MB}$ & $800 * 600$ & 98.068 \\
\hline & & & & $\mathrm{db} 4$ & $27.2 \mathrm{~KB}$ & $1.38 \mathrm{MB}$ & $802 * 602$ & 98.06 \\
\hline & & & & $\mathrm{db} 5$ & $26.5 \mathrm{~KB}$ & $1.37 \mathrm{MB}$ & $802 * 602$ & 98.111 \\
\hline
\end{tabular}




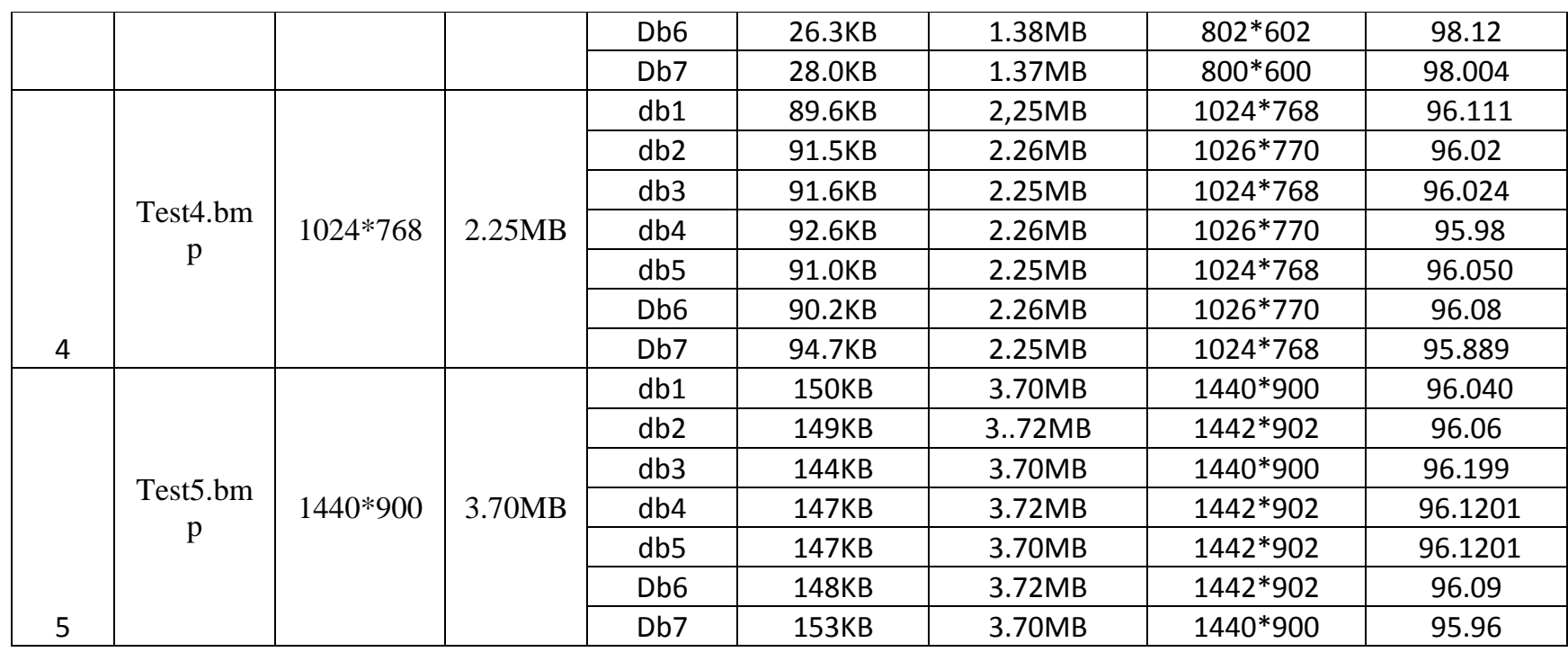

Table 5: Compression Analysis for wavelets family 'rbio'

\begin{tabular}{|c|c|c|c|c|c|c|c|c|}
\hline $\begin{array}{l}\text { SN. } \\
\text { O }\end{array}$ & $\begin{array}{l}\text { Input } \\
\text { Image }\end{array}$ & Dimension & $\begin{array}{l}\text { Original } \\
\text { Size }\end{array}$ & Wavelets & $\begin{array}{l}\text { Compressed } \\
\text { Size }\end{array}$ & $\begin{array}{l}\text { Decompressed } \\
\text { Size }\end{array}$ & $\begin{array}{l}\text { Decompressed } \\
\text { Dimension }\end{array}$ & $\begin{array}{l}\text { Compression } \\
\text { Ratio }\end{array}$ \\
\hline \multirow{3}{*}{1} & \multirow{3}{*}{ Test1.bmp } & \multirow{3}{*}{$320 * 240$} & \multirow{3}{*}{$225 \mathrm{~KB}$} & Rbio1.1 & $11.2 \mathrm{~KB}$ & $225 \mathrm{~KB}$ & $320 * 240$ & 95.0222 \\
\hline & & & & Rbio1.3 & $11.5 \mathrm{~KB}$ & $225 \mathrm{~KB}$ & $320 * 240$ & 94.888 \\
\hline & & & & Rbio1.5 & $11.6 \mathrm{~KB}$ & $225 \mathrm{~KB}$ & $320 * 240$ & 94.844 \\
\hline \multirow[b]{3}{*}{2} & \multirow{3}{*}{ Tes2.bmp } & \multirow{3}{*}{$512 * 512$} & \multirow{3}{*}{$768 \mathrm{~KB}$} & Rbio1.1 & $22.3 \mathrm{~KB}$ & $768 \mathrm{~KB}$ & $512 * 512$ & 97.096 \\
\hline & & & & Rbio1.3 & $23.4 \mathrm{~KB}$ & $768 \mathrm{~KB}$ & $512 * 512$ & 96.953 \\
\hline & & & & Rbio1.5 & $22.4 \mathrm{~KB}$ & $768 \mathrm{~KB}$ & $512 * 512$ & 97.083 \\
\hline \multirow[b]{3}{*}{3} & \multirow{3}{*}{ Test3.bmp } & \multirow{3}{*}{$800 * 600$} & \multirow{3}{*}{$1.37 \mathrm{MB}$} & Rbio1.1 & $27.1 \mathrm{~KB}$ & $1.37 \mathrm{MB}$ & $800 * 600$ & 98.068 \\
\hline & & & & Rbio1.3 & $26.7 \mathrm{~KB}$ & $1.37 \mathrm{MB}$ & $800 * 600$ & 98.096 \\
\hline & & & & Rbio1.5 & $26.1 \mathrm{~KB}$ & $1.37 \mathrm{MB}$ & $800 * 600$ & 98.139 \\
\hline \multirow[b]{3}{*}{4} & \multirow{3}{*}{ Test4.bmp } & \multirow{3}{*}{$1024 * 768$} & \multirow{3}{*}{$2.25 \mathrm{MB}$} & Rbio1.1 & 89.7KB & $2.25 \mathrm{MB}$ & $1024 * 768$ & 96.040 \\
\hline & & & & Rbio1.3 & $88.6 \mathrm{~KB}$ & $2.25 \mathrm{MB}$ & $1024 * 768$ & 96.199 \\
\hline & & & & Rbio1.5 & $88.4 \mathrm{~KB}$ & $2.25 \mathrm{MB}$ & $1024 * 768$ & 96.093 \\
\hline \multirow[b]{3}{*}{5} & \multirow{3}{*}{ Test5.bmp } & \multirow{3}{*}{$1440 * 900$} & \multirow{3}{*}{$3.70 \mathrm{MB}$} & Rbio1.1 & $150 \mathrm{~KB}$ & 3.70MB & $1440 * 900$ & 96.04 \\
\hline & & & & Rbio1.3 & $144 \mathrm{~KB}$ & 3.70MB & $1440 * 900$ & 96.19 \\
\hline & & & & Rbio1.5 & 148KB & 3.70MB & $1440 * 900$ & 96.09 \\
\hline
\end{tabular}

Table6: Compression Analysis for wavelets family 'sym'

\begin{tabular}{|c|c|c|c|c|c|c|c|c|}
\hline SN.O & $\begin{array}{l}\text { Input } \\
\text { Image }\end{array}$ & Dimension & $\begin{array}{l}\text { Original } \\
\text { Size }\end{array}$ & Wavelets & $\begin{array}{l}\text { Compressed } \\
\text { Size }\end{array}$ & $\begin{array}{l}\text { Decompressed } \\
\text { Size }\end{array}$ & $\begin{array}{l}\text { Decompressed } \\
\text { Dimension }\end{array}$ & $\begin{array}{l}\text { Compression } \\
\text { Ratio }\end{array}$ \\
\hline \multirow{3}{*}{1} & \multirow{3}{*}{ Test1.bmp } & \multirow{3}{*}{$320 * 240$} & \multirow{3}{*}{$225 \mathrm{~KB}$} & sym2 & $11.5 \mathrm{~kb}$ & $228 \mathrm{~kb}$ & $322 * 242$ & 94.88 \\
\hline & & & & sym3 & $11.8 \mathrm{~kb}$ & $225 \mathrm{~kb}$ & $320 * 240$ & 95.755 \\
\hline & & & & sym4 & $12.5 \mathrm{~kb}$ & $228 \mathrm{~kb}$ & $322 * 242$ & 94.444 \\
\hline \multirow[b]{3}{*}{2} & \multirow{3}{*}{ Tes2.bmp } & \multirow{3}{*}{$512 * 512$} & \multirow{3}{*}{$768 \mathrm{~KB}$} & sym2 & $22.7 \mathrm{~kb}$ & $775 \mathrm{~kb}$ & $514 * 514$ & 97.04 \\
\hline & & & & sym3 & $24.0 \mathrm{~kb}$ & $768 \mathrm{~kb}$ & $512 * 512$ & 96.875 \\
\hline & & & & sym4 & $24.3 \mathrm{~kb}$ & $775 \mathrm{~kb}$ & $514 * 514$ & 96.83 \\
\hline \multirow[b]{3}{*}{3} & \multirow{3}{*}{ Test3.bmp } & \multirow{3}{*}{$800 * 600$} & \multirow{3}{*}{$1.37 \mathrm{MB}$} & sym2 & $26.1 \mathrm{~kb}$ & $1.38 \mathrm{mb}$ & $802 * 602$ & 98.13 \\
\hline & & & & sym3 & $27.1 \mathrm{~kb}$ & $1.37 \mathrm{mb}$ & $800 * 600$ & 98.068 \\
\hline & & & & sym4 & $27.0 \mathrm{~kb}$ & $1.38 \mathrm{mb}$ & $802 * 602$ & 98.07 \\
\hline \multirow[b]{3}{*}{4} & \multirow{3}{*}{ Test4.bmp } & \multirow{3}{*}{$1024 * 768$} & \multirow{3}{*}{$2.25 \mathrm{MB}$} & sym2 & $91.5 \mathrm{~kb}$ & $2.26 \mathrm{mb}$ & $1026 * 770$ & 96.02 \\
\hline & & & & sym3 & $91.9 \mathrm{~kb}$ & $2.25 \mathrm{mb}$ & $1024 * 768$ & 96.011 \\
\hline & & & & sym4 & $93.5 \mathrm{~kb}$ & $2.26 \mathrm{mb}$ & $1026 * 770$ & 95.94 \\
\hline \multirow[b]{3}{*}{5} & \multirow{3}{*}{ Test5.bmp } & \multirow{3}{*}{$1440 * 900$} & \multirow{3}{*}{$3.70 \mathrm{MB}$} & sym2 & $149 \mathrm{~kb}$ & $3.72 \mathrm{mb}$ & $1442 * 902$ & 96.06 \\
\hline & & & & sym3 & $144 \mathrm{~kb}$ & $3.70 \mathrm{mb}$ & $1440 * 900$ & 96.199 \\
\hline & & & & sym4 & $149 \mathrm{~kb}$ & $3.72 \mathrm{mb}$ & $1442 * 902$ & 96.06 \\
\hline
\end{tabular}


Table 7: Compression Analysis for wavelets family 'haar'

\begin{tabular}{|c|l|l|l|l|l|l|l|l|}
\hline $\begin{array}{l}\text { SN. } \\
\text { O }\end{array}$ & $\begin{array}{l}\text { Input } \\
\text { Image }\end{array}$ & $\begin{array}{l}\text { Dimensio } \\
\text { n }\end{array}$ & $\begin{array}{l}\text { Origina } \\
\text { I Size }\end{array}$ & $\begin{array}{l}\text { Wavelet } \\
\text { s }\end{array}$ & $\begin{array}{l}\text { Compresse } \\
\text { d Size }\end{array}$ & $\begin{array}{l}\text { Decompresse } \\
\text { d Size }\end{array}$ & $\begin{array}{l}\text { Decompresse } \\
\text { d Dimension }\end{array}$ & $\begin{array}{l}\text { Compressio } \\
\text { n Ratio }\end{array}$ \\
\hline 1 & $\begin{array}{c}\text { Test1.bm } \\
\text { p }\end{array}$ & $320 * 240$ & $225 \mathrm{~KB}$ & Haar & $11.2 \mathrm{~kb}$ & $225 \mathrm{~kb}$ & $320 * 240$ & 95.022 \\
\hline 2 & $\begin{array}{c}\text { Tes2.bmp } \\
\text { Test3.bm } \\
\mathrm{p}\end{array}$ & $512 * 512$ & $760 \mathrm{~KB}$ & Haar & $43.0 \mathrm{~kb}$ & $768 \mathrm{~kb}$ & $512 * 512$ & 94.40 \\
\hline 3 & $\begin{array}{c}1.37 \mathrm{MB} \\
\text { haar }\end{array}$ & $51.8 \mathrm{~kb}$ & $1.37 \mathrm{mb}$ & $800 * 600$ & 96.307 \\
\hline 4 & $\begin{array}{c}\text { Test4.bm } \\
\mathrm{p}\end{array}$ & $1024 * 768$ & $2.25 \mathrm{MB}$ & Haar & $141 \mathrm{~kb}$ & $2.25 \mathrm{mb}$ & $1024 * 768$ & 93.880 \\
\hline 5 & $\begin{array}{c}\text { Test5.bm } \\
\mathrm{p}\end{array}$ & $1440 * 900$ & $3.70 \mathrm{MB}$ & haar & $240 \mathrm{~kb}$ & $3.70 \mathrm{mb}$ & $1440 * 900$ & 93.665 \\
\hline
\end{tabular}

Table8: Compression Analysis for wavelets family 'dmey'

\begin{tabular}{|c|l|l|l|l|l|l|l|l|}
\hline $\begin{array}{l}\text { SN. } \\
\text { O }\end{array}$ & $\begin{array}{l}\text { Input } \\
\text { Image }\end{array}$ & $\begin{array}{l}\text { Dimensio } \\
\text { n }\end{array}$ & $\begin{array}{l}\text { Origina } \\
\text { I Size }\end{array}$ & $\begin{array}{l}\text { Wavelet } \\
\mathbf{s}\end{array}$ & $\begin{array}{l}\text { Compresse } \\
\text { d Size }\end{array}$ & $\begin{array}{l}\text { Decompresse } \\
\text { d Size }\end{array}$ & $\begin{array}{l}\text { Decompresse } \\
\text { d Dimension }\end{array}$ & $\begin{array}{l}\text { Compressio } \\
\text { n Ratio }\end{array}$ \\
\hline 1 & $\begin{array}{c}\text { Test1.bm } \\
\text { p }\end{array}$ & $320 * 240$ & $225 \mathrm{~KB}$ & dmey & $28.6 \mathrm{~kb}$ & $225 \mathrm{~kb}$ & $320 * 240$ & 87.288 \\
\hline 2 & Tes2.bmp & $512 * 512$ & $768 \mathrm{~KB}$ & Dmey & $43.0 \mathrm{~kb}$ & $768 \mathrm{~kb}$ & $512 * 512$ & 94.401 \\
\hline 3 & $\begin{array}{c}\text { Test3.bm } \\
\mathrm{p}\end{array}$ & $800 * 600$ & $1.37 \mathrm{MB}$ & Dmey & $51.8 \mathrm{~kb}$ & $1.37 \mathrm{mb}$ & $800 * 600$ & 96.307 \\
\hline 4 & $\begin{array}{c}\text { Test4.bm } \\
\mathrm{p}\end{array}$ & $1024 * 768$ & $2.25 \mathrm{MB}$ & Dmey & $141 \mathrm{~kb}$ & $2.25 \mathrm{mb}$ & $1024 * 768$ & 93.880 \\
\hline 5 & $\begin{array}{c}\text { Test5.bm } \\
\mathrm{p}\end{array}$ & $1440 * 900$ & $3.70 \mathrm{MB}$ & dmey & $240 \mathrm{~kb}$ & $3.70 \mathrm{mb}$ & $1440 * 900$ & 93.665 \\
\hline
\end{tabular}

Here input images set divided into five categories on the basis of size of input data and extract best two wavelets family from the above result on the basis of compression ratio in percentage. e.g. for Test1.bmp image (Size 225kb) four wavelets with first rating bior1.1, rbio1.1,haar, and Daubechies1give same compression ratio i.e. $95.0222 \%$ and one wavelets with second rating Coif 2 gives less compression ratio $94.93 \%$.

\section{CONCLUSION}

From the above table conclude the best compression ratio among the wavelets Family is mention below five different range of input image.

\begin{tabular}{|c|c|c|}
\hline Size & Best wavelets & $\begin{array}{c}\text { Compression } \\
\text { Ratio (in \%) }\end{array}$ \\
\hline \multirow{2}{*}{ Under 500 KB } & $\begin{array}{c}\text { bior1.1, rbio1.1,haar, } \\
\text { Db1 }\end{array}$ & $95.0222 \%$ \\
\cline { 2 - 3 } & Coif2 & $94.93 \%$ \\
\hline $501 \mathrm{~KB}-$ & $\mathrm{db} 1$, bior1.1,rbio1.1 & $97.096 \%$ \\
\cline { 2 - 3 } $1024 \mathrm{~KB}$ & Coif2 & $97.08 \%$ \\
\hline 1025KB-1.5MB & Coif 2 & 96.199 \\
\cline { 2 - 3 } & $\mathrm{db} 2$, sym2,rbio1.5 & $98.15 \%$ \\
\hline 1.51MB-2.5MB & rbio 1.5 & 96.163 \\
\cline { 2 - 3 } & rbio 1.3 & $96.15 \%$ \\
\hline $2.51 \mathrm{MB}-$ & rbio 1.3, sym 3, db3 & $96.199 \%$ \\
\cline { 2 - 3 } $4.5 \mathrm{MB}$ & Db4,db5 & $96.12 \%$ \\
\hline
\end{tabular}

On the basic of size of input image select the best compression mechanism with particular wavelets from the above table. Here after execution above said algorithms point out one more important mechanism in the research with image compression is that apart from above listed wavelets some wavelets are not supporting with compression, it is also verified from experimental aspects with the help of above said algorithms.
List of wavelets which are not supporting

\begin{tabular}{|l|l|l|l|}
\hline Meyr & gaus1 & Mexh & Merl \\
\hline Shan & Fbsp & Cmor & \\
\hline
\end{tabular}

In order to decrease the cost of storage, researchers have emphasized and shown importance to optimization techniques with wavelets. There are many techniquesfor waveletstransformation but their main focus is on mixed transformation using different wavelets family. And analysis the quality of images on the basic of some parameter like PSNR, MSE, NCC, and CR, and also conclude that which wavelets family member are better with respect to different quality parameter.

\section{REFERENCES}

[1] Jerome M. Sharpiro, "Embedded Image Coding Using Zerotrees Of Wavelet Coefficients" IEEE Transactions On Signal Processing. Vol.41. No.12, December 1993.

[2] Andrew B. Watson NASA Ames Research Center "Image Compression Using The Discrete Cosine Transform" Mathematica Journal, 4(1), 1994, p. 81-88.

[3] Guilherme Cardoso and Jafar Saniie "Performance Evaluation of DWT, DCT and WHT for Compression of Ultrasonic Signals" 2004 IEEE Transaction International Ultrasonics, Ferroelectrics, and Frequency Control Joint 50th Anniversary Conference.

[4] Suchitra Shrestha and Khan Wahid "Hybrid DWT-DCT Algorithm For Biomedical Image And Video Compression Applications, 2010" IEEE Transaction 10th International Conference on Information Science, Signal Processing and their Applications. (ISSPA 2010) 
[5] Archana Deshlahra, G. S. Shirnevar, Dr. A. K. Sahoo "A Comparative Study of DCT, DWT \&Hybrid (DCTDWT) Transform"2010 IEEE.

[6] Aree Ali Mohammed and Jamal Ali Hussian "Hybrid Transform Coding Scheme for Medical Image Application" 2011 IEEE Transaction.

[7] Smitha Joyce Pinto, Prof. Jayanand P. Gawande" Performance Analysis of Medical Image Compression Techniques" 2012 IEEE Transaction.

[8] D. Malarvizhi , Dr. K. Kuppusamy "A New Entropy Algorithm for Image Compression Using DCT" International Journal of Engineering Trends and Technology- Volume3Issue3- 2012

[9] Er. RamandeepKaur,NavneetRandhawa "Image Compression Using Discrete Cosine Transform \&Discrete Wavelet Transform" International Journal of Computing \& Business Research ISSN (Online): 22296166.

[10] Nikita Bansal, Sanjay Kumar Dubey "Image Compression Using Hybrid Transform Technique" Journal of Global Research in Computer Science, 4 (1), January 2013, 13-17.

[11] Manjinder Kaur, Gaganpreet Kaur "Survey of Lossless and Lossy Image Compression Techniques" International Journal of Advanced Research in Computer Science and Software Engineering 3(2), February - 2013, pp. 323326.

[12] Akshay Kekre , Dr. Sanjay Pokle "Improved Image Compression Using Wavelet Transform and Differential Pulse Code Modulation Technique" International Journal of Engineering Research \& Technology (IJERT) Vol. 2 Issue 7, July - 2013.
[13] Neelesh Kumar Sahu,ChandrashekharKamargaonkar "A Survey on Various Medical Image Compression Techniques" International Journal of Science, Engineering and Technology Research (IJSETR) Volume 2, Issue 2, February 2013.

[14] NavpreetSaroya,PrabhpreetKaur "Analysis Of Image Compression Algorithm Using DCT And DWT Transforms" International Journal of Advanced Research in Computer Science and Software Engineering 4(2), February - 2014, pp. 897-900.

[15] R. Bhavithra, L. AyeeshaBegame, K.S.L. Deepika "A Survey on Medical Image Compression Based on Transform "SSRG International Journal of Electronics and Communication Engineering (SSRG-IJECE) volume1 issue6 August 2014.

[16] MalvikaDixit,Harbinder Singh “An Enhanced Hybrid Technology for Digital Image Compression "SSRG International Journal of Electronics and Communication Engineering (SSRG-IJECE) - volume1 issue7 Sep 2014.

[17] MahinderpalSingh,MeenakshiGarg "Mixed DWT-DCT Approached Based Image Compression Technique" International Journal Of Engineering And Computer Science ISSN: 2319-7242 Volume 3 Issue 11 November, 2014 Page No. 9008-9111.

[18] K. Ayyappa Swamy , C. Somasundar Reddy, K. Durga Sreenivas , Image Compression Using Hybrid DCTDWT Transform, Volume 5, Issue 5, May 2015 ISSN: $2277128 \mathrm{X}$

[19] Kumar, T. and K. Verma, 2010a. A theory based on conversion of RGB image to gray image. Int. J. Computer. Appli., 7: 5-12. DOI: 10.5120/1140-1493. 